\title{
A prize, an award and Christmas crackers: season's greetings from the MJA
}

\author{
Nicholas J Talley, on behalf of the MJA editorial team
}

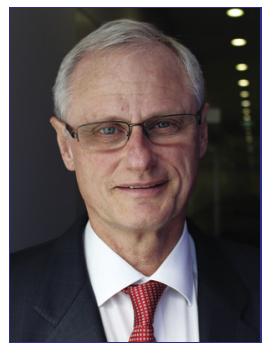

W

elcome to the Christmas issue of the $M J A$, an annual tradition in which we publish unusual, witty, funny, and even astounding images, stories and non-serious studies in our "Christmas crackers" competition. All articles entered for this competition have undergone our usual rigorous publication process, and the best submission, as judged by our entire editorial team, wins a Christmas hamper. You may muse about the diagnosis for Santa's red cheeks ${ }^{1}$ or chuckle at the collective nouns for your colleagues' specialties (perhaps even your own); ${ }^{2}$ you might be enlightened by the thought of diagnosing Nutcracker syndrome, ${ }^{3}$ or, if Tchaikovsky is not your beat, the effects of AC/DC on your surgical skills; ${ }^{4}$ while if you are saddened by a rejected grant application, reading the Journal's most recent love letter may strike a chord. And the less said about hot dogs ... ${ }^{6}$ The national and international interest in this light-hearted competition remains high; we thank everyone who contributed, and only regret we could not publish every entry. This year we are delighted to announce that the joint winners of our competition are Fancourt and her co-authors ${ }^{4}$ and Rouse and Radigan, ${ }^{6}$ while Ge and her colleagues achieved a distinguished third place. ${ }^{1}$

One of the missions of the MJA is to publish excellent original research that will positively change clinical practice or health policy. We particularly thank all of the dedicated MJA peer reviewers of the past year (listed on pages 496-497); we all recognise that you provide an essential contribution to the progress of medical science. All research published in the MJA undergoes blinded peer review, as well as detailed editorial and statistical reviews. We reject about $85 \%$ of original research submissions (Box), and authors who have achieved publication in the Journal can be assured that their work is highly regarded by their peers. Research published in the MJA has a national impact, and this impact is one of the metrics for our journal we will be monitoring in the future. The online version of the Journal is increasingly read internationally, with overseas "hits" at the MJA website now providing almost one-quarter of the enormous traffic to the site. Our primary goal is always excellence: to assist authors to present their data so that it has the greatest impact, and to help disseminate their findings widely in print, online, and through social media.

The 2016 MJA-MDA National Prize for Excellence in Medical Research, worth $\$ 10000$, will honour the authors of the best original clinical research article published by the MJA during the past year. The Journal editors will prepare a shortlist of the very best research published in 2016, as measured by five criteria: significance, novelty, study quality, research effort, and presentation quality. The MJA Expert Advisory Group, a panel of leading researchers and clinicians from around Australia and the world, will independently select the winner during the first quarter of 2017, and the prize will be awarded at the national

\begin{tabular}{|lccc|}
\hline \multicolumn{4}{c}{ Manuscripts received, 1 July 2015 - 30 June 2016} \\
& Received & Accepted & Accepted (\%) \\
\hline All articles & 1407 & 375 & $27 \%$ \\
Research & 424 & 59 & $14 \%$ \\
Case reports & 221 & 28 & $13 \%$ \\
Editorials & 49 & 41 & $84 \%$ \\
Perspectives & 170 & 72 & $42 \%$ \\
Letters & 49 & 39 & $80 \%$ \\
Short reports & 114 & 41 & $36 \%$ \\
\hline Mean days to decision & & All articles & Research articles \\
To accept & & 63 & 108 \\
To reject & & 29 & 34 \\
Number of reviewers & & & 1038 \\
\end{tabular}

conference of the Australian Medical Association in May 2017. We wish every author who has published a research article in the MJA during 2016 the very best during judging for the 2016 prize.

In preparation for this final issue of the year, the $M J A$ editors have selected examples of the research we have published during 2016 that were highlights with respect to the breadth and depth of research excellence, and because the findings have important implications for health practice or policy.

Starting with cardiovascular disease: heart failure is still a leading cause of death in Australia. Newton and his colleagues ${ }^{7}$ observed that evidence-based therapies are underutilised despite several drugs having been established as being effective in patients with heart failure, and that there is wide variation in the length of hospitalisation for patients in different locations. Guidelines alone are not sufficient for driving effective practice change; concerted leadership from relevant experts is needed to optimise therapy for heart failure patients across the system and to improve their quality of life.

Exciting advances in treating cardiac disease were reported by Khan and his co-authors in their report ${ }^{8}$ about the remarkable impact of pre-hospital thrombolysis on outcomes for patients with ST-elevation (STEMI) myocardial infarctions in a New South Wales region (Hunter-New England) where large distances impede access to top quality treatment facilities. This is a model of care that should be adopted by other regions where timely primary percutaneous coronary intervention is not available for patients with a STEMI.

We know that sepsis can present very subtly, but also that it is still associated with high mortality. Burrell and his colleagues ${ }^{9}$ provided evidence that the SEPSIS KILLS program, implemented in 97 emergency departments across NSW, is reducing mortality, time in intensive care, and length of hospital stay for patients. The 
implication is that the program should be rolled out in all Australian hospitals, and we look forward to updates on its implementation.

Turning to cancer research, Sampurno and her co-authors ${ }^{10}$ reported important findings about outcomes for patients receiving prostate cancer care in Victoria. This is a controversial area, especially because prostate-specific antigen (PSA) testing in healthy older men remains prevalent. Encouragingly, unnecessary active treatment of patients with low risk disease is decreasing in Victoria, and more appropriate treatment is being provided for men with high risk and metastatic disease. Overtreatment of men with positive PSA test results (and avoiding unnecessary testing) remains a priority health issue in Australia.

Colon cancer is still a serious scourge in our country, despite screening. Boyce and colleagues ${ }^{11}$ reported that the incidence of young-onset colorectal cancer (in patients under 50 years of age) has increased recently in the United States and Europe, but not in NSW. Even so, clinicians need to be aware that $6 \%$ of colon cancers are diagnosed in younger people, so that alarm symptoms in this age group should not be ignored. Cancers in younger patients were more commonly located in the rectum, and were more advanced at diagnosis than in those over 50, although 5-year cancer-specific survival, as might be expected, was better than for older patients.

Evidence-based, optimal treatment of burns is important, but, as Cleland and her co-authors ${ }^{12}$ highlight, there is a dearth of evidence about best practice management, with relatively few high quality clinical trials having been undertaken. Their large study of registry data for 10 of the 12 adult burns units in Australasia identified wide variations in practice and patient outcomes, suggesting there is significant scope for improvement by implementing practices supported by best evidence.

Finally, targets in the emergency department (ED) remain both political and health care problems in 2016. Sullivan and colleagues $^{13}$ conducted a retrospective study of 59 Australian EDs, with 12.5 million episodes of ED care, and found that inhospital mortality for emergency admissions decreased as compliance with the national emergency access target (NEAT) improved - until NEAT compliance reached $83 \%$, beyond which the inverse linear relationship between mortality and NEAT compliance was lost. While this has implications for health policy, and some may argue that it vindicates the use of the NEAT, a cause-and-effect relationship cannot be established at the present time.

These and other studies will be considered for the MJA-MDA National Prize for Excellence in Medical Research, and we welcome your views about these and the other articles we publish. As part of our quality process, we have re-instated a vigorous
Letters to the Editor section for the best correspondence we receive, but we also have an online forum for your immediate comments. We wish to ensure that any errors in our research articles that have been overlooked, despite our best efforts, are exposed, discussed and, where appropriate, corrected.

In 2017, you can expect further evolution of your Journal. We continue to welcome your feedback on all our articles and your ideas for new content.

From all of us here at the $M J A$, we wish you and your family a safe, healthy and joyous holiday season, and we look forward to welcoming back all of our readers, authors and reviewers in 2017.

\section{Competing interests: No relevant disclosures.}

(c) 2016 AMPCo Pty Ltd. Produced with Elsevier B.V. All rights reserved.

1 Ge L, O'Connor AA, Polcz MM, Sebaratnam DF. A cheeky diagnosis. Med J Aust 2016; 205: 520

2 Massie RJH. Medical collective nouns. Med J Aust 2016; 205: 527.

3 Epstein RJ. Be careful what you wish for. Med J Aust 2016; 205: 521-522.

4 Fancourt D, Burton TM, Williamon A. The razor's edge: Australian rock music impairs men's performance when pretending to be a surgeon. Med J Aust 2016; 205: 515-518.

5 McGuane JT. It's not me, it's you: why l'm breaking up with medical research. Med J Aust 2016; 205: 525-526.

6 Rouse T, Radigan J. What's in your hot dog? A histological comparative analysis. Med J Aust 2016; 205: 519-520.

7 Newton PJ, Davidson PM, Reid CM, et al. Acute heart failure admissions in New South Wales and the Australian Capital Territory: the NSW HF Snapshot Study. Med J Aust 2016; 204: 113. https://www.mja.com.au/journal/2016/204/3/acute-heart-failureadmissions-new-south-wales-and-australian-capital-territory

8 Khan AA, Williams T, Savage L, et al. Pre-hospital thrombolysis in ST-segment elevation myocardial infarction: a regional Australian experience. Med J Aust 2016; 205: 121-125. https://www.mja.com.au/journal/2016/205/3/pre-hospital-thrombolysis-st-segmentelevation-myocardial-infarction-regional

9 Burrell AR, McLaws M, Fullick M, et al. SEPSIS KILLS: early intervention saves lives. Med J Aust 2016; 204: 73. https://www.mja.com.au/journal/2016/204/2/sepsis-killsearly-intervention-saves-lives

10 Sampurno F, Earnest A, Kumari PB, et al. Quality of care achievements of the Prostate Cancer Outcomes Registry-Victoria. Med J Aust 2016; 204: 319. https://www.mja.com.au/ journal/2016/204/8/quality-care-achievements-prostate-cancer-outcomes-registry-victoria

11 Boyce S, Nassar N, Lee C, et al. Young-onset colorectal cancer in New South Wales: a population-based study. Med J Aust 2016; 205: 465-470. https://www.mja.com.au/ journal/2016/205/10/young-onset-colorectal-cancer-new-south-wales-populationbased-study

12 Cleland H, Greenwood JE, Wood FM, et al. The Burns Registry of Australia and New Zealand: progressing the evidence base for burn care. Med J Aust 2016; 204: 195. https:// www.mja.com.au/journal/2016/204/5/burns-registry-australia-and-new-zealandprogressing-evidence-base-burn-care

13 Sullivan C, Staib A, Khanna S, et al. The National Emergency Access Target (NEAT) and the 4-hour rule: time to review the target. Med J Aust 2016; 204: 354. https://www.mja. com.au/journal/2016/204/9/national-emergency-access-target-neat-and-4-hour-ruletime-review-target

\section{The MJA Editorial Advisory Committee}

\section{Ms Elise Buisson}

Dr Jonathan Christiansen

Associate Professor Flavia M Cicuttini

Professor Jane Dacre

Professor Allan Fels

Professor Peter G Gibson

Professor Marc Gladman
Dr Charles Guest

Professor Claire L Jackson

Dr Zsuzsoka Kecskes

Associate Professor Kelvin M Kong

Professor Christopher Levi

Professor Jennifer Martin

Professor Patrick D McGorry
Professor Paul Moayyedi

Professor lan N Olver

Professor Joseph J Y Sung

Dr Catherine Yelland

Professor Neville D Yeomans

Professor Jeffrey Zajac 
Reviewers (submitted between 31/10/2015 and 1/11/2016)

Penelope A Abbott

Sarah J Abrahamson

Michael J Abramson

Stephen P Ackland

Jason P Acworth

Susan E Adams

Rachael Addicott

Barbara-Ann Adelstein

Stephen Adelstein

Michael A Adena

Robert G Adler

Meera R Agar

Peter Aitken

Philip D Aitken

Karyn E Alexander

Charles Algert

Sonia Allan

Roger W G Allison

Stephen Allison

Anthony M Allworth

Marlies Alvarenga

John V Amerena

Janaki Amin

Lisa H Ami

Craig S Anderson

Warwick P Anderson

David Andresen

Gavin Andrews

Jane M Andrews

Mark N Appleyard

John S Archer

Nigel R Armfield

Bruce K Armstrong

Ruth M Armstrong

Constantine N Aroney

Peter Arvier

Michael A Ashby

Eugene Athan

John J Atherton

David N Atkinson

$\mathrm{R}$ Leigh Atkinson

Philip E G Aylward

Oyekoya T Ayonrinde

Peter D Baade

Tim Badgery-Parker

Chris Baggoley

Peter A Baghurst

Paul M Bailey

Ross S Bailie

Rob Baird

Ross I Baker

Eileen Baldry

Mohammed S Ballal

Zsolt J Balogh

Lilon G Bandler

Neil Banham

Josephine Barbaro

Lesley Barclay

Amanda Barnard

David J Barnes

Harold Bartlett

Robert G Batey

Marijka Batterham

Philip J. Batterham

Paul A Bauert

Adrian E Bauman

Peter E Baume

Frank H Beard

Peter J Beaumont

Kerri Beckmann

Justin J Beilby

Anthony Jenner Bell

James R Bell

Derrick A Bennett

Jill Benson

Roy G Beran

Lisa Bero

Martin Berry

Andrew D Bersten

$\mathrm{J} \mathrm{H}$ Nicholas Bett

Baki Billah

Frances A Birrell
Roderick O Bishop

Marie M Bismark

Deborah A Black

Kirsten I Black

David J Blacker

Christopher F Bladin

Ilse Blignault

C Leigh Blizzard

Mark Bloch

Peter A Blombery

Neville Board

Felix Bochner

Nikolai Bogduk

Michael D Bollen

Stephen N Bolsin

Patrick G M Bolton

David Bolzonello

Michael A Bonning

Michael J Bourke

Christopher P Bourne

Craig S Boutlis

Francis J Bowden

Simon D Bowler

Anne-Marie Boxall

Steven C Boyages

Ian W Boyd

Frances M Boyle

Michael J Boyle

Clare E Bradley

George Braitberg

Jeffrey Braithwaite

Annette J Braunack-Mayer

Bruce J Brew

David B Brieger

Jo-anne E Brien

Esther Brigant

Julie K Brimblecombe

Fraser J Brims

Peter J Bristow

Helena C Britt

Philip N Britton

Simon Andrew Broadley

Wendy Elizabeth Brodribb

Julia M L Brotherton

Lona C Brown

Natasha Jane Brown

Lois H Browne

Elizabeth Buckley

John F Buckley

Nicholas A Buckley

Kirsty L Buising

Michael D Buist

Chris Bullen

Alexandra J C Bune

Jonathan G W Burdon

John R Burgess

Bryan H Burmeister

John R Burnett

Colin D Butler

Phyllis N Butow

Joshua Byrnes

Petra T Bywood

John F Cade

Dominique A Cadilhac

J William F Cairns

Adrian J Cameron

Donald J S Cameron

Ian D Cameron

Peter A Cameron

W Ian Cameron

David G Campbell

Marilyn A Campbell

Patricia Campbell

Terence J Campbell

Ben J Canny

Gideon A Caplan

Susan M Carden

Magnolia Cardona-Morrell

John B Carlin

Bronwyn E Carson

Phillip J Carson

Jonathan Carter
Judith Cashmore

Armand Casolin

Albert K F Chan

Elsie Chan

Raymond C Chan

Sally Chan

Andrew M Chanen

Karen Charlton

Mark D. Chatfield

Celia S-W Chen

Jack Chen

Stanley E-Hon Chen

Ian R Cheong

N Wah Cheung

Derek P Chew

Jun Chih

Jacqueline $\mathrm{H}$ Chirgwin

James Y J Choi

Catherine L Chojenta

Patty Chondros

Christopher Y P Choong

Eric Pui Fung Chow

Wojciech Chrzanowski

Leonid Churilov

Flavia M Cicuttini

Kathleen F Clapham

Caroline F Clarke

Marilyn J Clarke

Stephen J Clarke

Moira A Clay

Anton C Clifford

Peter M Clifton

Alan R Clough

Nicholas R Coatsworth

Tina Cockburn

Milton Cohen

Enrico W Coiera

Paul B Colditz

Justin J Coleman

Peter J Collignon

David M Colquhoun

Deborah J Colville

Elizabeth J Comino

John R Condon

Katherine M Conigrave

Christine Connors

Matthew C Cook

Nicholas B Cooling

Alan J Cooper

Michael D Coory

William Coote

Jan Copeland

Charles F Corke

Chris Coulter

Brendon J Coventry

Terry J Coyne

Maria E Craig

Susanna Cramb

Helen M Creasey

Julia L Crilly

David B Cross

Gary Crosthwaite

Brendan Crotty

Philip Crowley

Robert G Cumming

Margaret C Cummings

Bart J Currie

David C Currow

Jackie E Curtis

Melville J Da Cruz

Eleonora Dal Grande

Andrew Dalton

Seamus E Dalton

E R David Dammery

Scott K D'Amours

Mark D Daniell

Anthony M Dart

Mike Daube

A James M Daveson

Sandra K Davidson

Jane Davies

Lucy Davies

Elizabeth Ann Davis

lan D Davis

Richard O Day

John F de Campo

Caroline M de Costa

Julien $\mathrm{P}$ de Jager

Nicholas H de Klerk

Evelyne de Leeuw

Rachel Dear

Shelley Deeks

Christopher B Del Mar

Geoff Delaney

Martin B Delatycki

Stephen A Della-Fiorentina

Michael C d'Emden

Apo Demirkol

Justin T Denholm

Graeme J Dennerstein

A Robert Denniss

Karen M Detering

Peter G Devitt

Mirella Di Benedetto

Adrian Di Bisceglie

James A Dickinson

Rodney Dilley

Timothy A Dobbins

Annette J Dobson

Xenia Dolja-Gore

Gregory J Dore

Kirsty Douglas

Paul J Douglas

Jo A Douglass

Robert P Dowsett

Nic T Droste

Stephen J Duckett

Patricia Dudgeon

Michael J Dudley

Johan A Duflou

Anne E Duggan

James A Dunbar

Shane R Durkin

John M Dwyer

Peter Dwyer

John R Dyer 
Mike P Jones

Nicholas Jones

Anthony F Jorm

Matthew D Jose

Johan D Jurgens

Peter Kam

John Tzu-Hsiu Kao

Jessica Kasza

Judith M Katzenellenbogen

Margaret P Kay

Joey M Kaye

Zsuzsoka Kecskes

Damien Kee

Pookong Kee

Marc J N C Keirse

Nicholas A Keks

John E Kellow

Anne-Maree Kelly

Robert I Kelly

Peter G Kerr

Alison M Kesson

Soo Keat Khoo

Monique Kilkenny

Susan W Kim

Richard King

Scott Kinlay

David W Kissane

Andrew W Knight

Jen Kok

Paul A Komesaroff

Srinivas Kondalsamy-

Chennakesavan

Alex Konstantatos

Robert J Kosky

Steven Kossard

Vicki Kotsirilos

Gabor T Kovacs

Vicki L Krause

Anthea S Krieg

Karolina Krysinska

Paul A Kubler

Dennis L Kuchar

Susan E Kurrle

Susan Elizabeth Kurrle

Maarit Laaksonen

Antony R A Lafferty

Kim Lai

Thao Lam

Tina Lam

Erika Langham

Andrew J Langley

Matthew M Large

Richard G Larkins

Edmund Lau

M Roger Laurent

Matthew G Law

Nathan Lawrentschuk

Jamie Layland

Estelle Lazer

Liana Leach

Karin Leder

Richard P Lee

Victor Lee

Vincent Lee

Sandra G Leggat

Peter A Leggat, AM

James Leigh

Gary M Leong

Florence Levy

Lucy N Lewis

Steven J Lewis

Joel Lexchin

$\mathrm{Mu} \mathrm{Li}$

Wenbin Liang

Belinda Liddell

Helena Liira

Björn Lindkvist

Richard I Lindley

Nicholas Lintzeris

Wendy L Lipworth

Mark Little

Michael Livingston

Helen Lloyd
Sing Kai Lo

Mark Lock

Bebe Loff

Richard K S Loh

David F M Looke

Charles W Lott

Julia M Lowe

Judy A Lowthian

Guy L Ludbrook

William (Bill) Lukin

Gary David Lum, AM

Kehui K Luo

Sharyn Lymer

Nigel Lyons

Zaza Lyons

Kristine Macartney

Graeme A Macdonald

Graham J Macdonald

Peter S MacDonald

Robert J M MacGinley

Andrew I Maclsaac

Dorothy E M Mackerras

Finlay A Macrae

Bill Madden

Richard Cawley Madden

Guy J Maddern

lan Maddocks

Parker J Magin

Dianna J Magliano

Graeme P Maguire

Sriram Mahadev

Donna B Mak

Allison Malcolm

George Jack Mangos

Linda Mann

Haider R Mannan

Darren R Mansfield

Panos G Maouris

Lynette M March

Peter G Markey

Tania Markovic

Guy B Marks

John E Marley

Roderick I Marshall

Roger J Marshall

Andrew J Martin

Jane E Martin

Paul T Martin

Danielle Mazza

Grant McArthur

Brian R McAvoy

Elizabeth Anne McCarthy

Sally M McCarthy

Brian C McCaughan

Geoffrey W McCaughan

Geoffrey J McColl

Joseph G McCormack

Michael McCready

Fiona J McDonald

Michael A McDonough

Heather J McElroy

Suzanne P McEvoy

Alexander C McFarlane

Andrew McGee

Neil W McGill

Patrick D McGorry

Treasure M McGuire

Ellen L McIntyre

Peter B Mclntyre

Diana R McKay

Robert S McKelvey

Scott McKenzie

Andrew J McLachlan

Hamish McManus

Donald McNeil

Elizabeth McVie

Graham N Meadows

Alan P Meagher

Jacqueline K Mein

Craig Mellis

Richard M Mendelson

Fiona K Mensah
Patricia Metcalf

Elasma Milanzi

J Alasdair Millar

Jeremy L Millar

Caroline Miller

Russell K Miller

Roger L Milne

David H Mitchell

Imogen Mitchell

Philip B Mitchell

Francis Mitrou

Mohammed M Mohsin

Lindsay C Mollison

Michael Montalto

Brett D Montgomery

Elizabeth M Moore

Kim L Moretti

Susan J Morgan

Peter Morley

Philip L P Morris

Robert F W Moulds

Wendy Muircroft

Raymond J Mullins

Arthur (Bill) W Musk

Stephen P Myers

Paul Myhill

Paul S Myles

Lucio Naccarella

Vasi Naganathan

Michael Nance

Louise M Nash

Mark R Nelson

Susan J Neuhaus

Peter W New

Jonathan W Newbury

David A Newby

Louise K Newman

Tony Newman

Hanh T T Ngo

Anh-Minh Nguyen

Cattram Nguyen

Tuan V Nguyen

Kathleen M Nicholls

Stephen Nicholls

Geoffrey C Nicholson

Antony Nocera

Christopher J Nolan

Anna K Nowak

Dianne L O'Connell

Catherine C O'Connor

Kerin O'Dea

Melissa O'Donnell

Maree O'Keefe

Peter O'Leary

Ian N Olver

John K Olynyk

Sean O'Neill

John W Orchard

Liliana Orellana

Peter K O'Rourke

Pamela Palasanthiran

Kathryn S Panaretto

Nirmala Pandeya

Gordon B Parker

William A Parsonage

Jennifer S Parsons

Dennis R Pashen

Mahomed Said Patel

Christine L Paul

Georgia A Paxton

Brian B Peat

Alexia S Pena

David G Penington

Andrew G Penman

Martin Pera

Vlado Perkovic

Andrew Perry

Andrew F Pesce

Alexandra Phelan

Jennifer Philip

Christine B Phillips

Richard K S Phoon

Perry Pickhardt

Avinesh Pillai

Peter I Pillans

Angie N Pinto

Leon Piterman

Peter G Pohlner

Christopher S Pokorny

Rene G Pols

Jeffrey J Post

Henry Miles Prince

Peter J Psaltis

Carolyn Quadrio

Julie A Quinlivan

Brendan Quinn

Janette C Radford

Janette M Randall

Melissa Raven

William D Rawlinson

Phillip Read

Stephen J Read

Georget Reaiche

Jennifer S Reath

Carole Reeve

Glenn EM Reeves

Annette Regan

Brian G Regan

Lionel I Reyftmann

Bernadette Joy Richards

Gary E Richardson

Geoffrey J Riley

Malcolm D Riley

Ian T Ring

Danny Rischin

Brett Ritchie

Darren M Roberts

Michael S Roberts

Colin F Robertson

Iain K Robertson

Kurt C Roberts-Thomson

Peter J Roberts-Thomson

Monica C Robotin

Stephen J Robson

Ian Rockett

Anthony Rodgers 